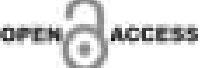

ATUALIZAÇÃO DE TEMA

\title{
Cirurgia Robótica no Tratamento Cirúrgico do Câncer de Próstata
}

\author{
Robotic Surgery in Surgical Treatment of Prostate Cancer
}

Correspondence addresses

Dr. Eduardo Café

eduardocafe@gmail.com

Received: July 29, 2019

Revised: August 26, 2019

Accepted: August 29, 2019

Published: September 27, 2019

Data Availability Statement: All relevant data are within the paper and its Supporting Information files.

Funding: This work was the result of author's initiative. There was no support of research or publication funds.

Competing interests: The author has declared that no competing interests exist.

\section{Copyright}

(C) 2019 by Santa Casa

de Misericórdia da Bahia.

All rights reserved.

ISSN: 2526-5563

\author{
Eduardo Café ${ }^{1}$ \\ ${ }^{1}$ Professor Adjunto Escola Bahiana de Medicina e Saúde Pública; Chefe do Departamento \\ de Cirurgia Hospital Santa Izabel; Salvador, Bahia, Brasil
}

Indivíduos diagnosticados com câncer de próstata muitas vezes devem fazer a escolha se irão se submeter ao tratamento cirúrgico ou não, uma vez que isto provavelmente irá influenciar na qualidade de suas vidas. Apesar de ser a principal forma de tratamento curativo para o câncer de próstata localizado, a prostatectomia radical pode impactar no controle do câncer e na função sexual e urinária do indivíduo. Em geral, os pacientes selecionam tratamentos menos mórbidos e minimamente invasivos desde que apresentem eficácias equivalentes. A prostatectomia radical robótica (PRR) representa o mais recente avanço no tratamento cirúrgico do câncer de próstata. $O$ tratamento se baseia na abordagem cirúrgica minimamente invasiva da prostatectomia laparoscópica, otimizada pela tecnologia robótica. A utilização de braços robóticos com instrumentos capazes de realizar movimentos com a mesma amplitude do punho humano, com filtro para tremores, associado a um sistema avançado de imagem tridimensional, permite ao cirurgião operar, dissecar, suturar com um refinamento de movimentos e qualidade de imagem sem precedentes. Estimativas atuais dão conta de que mais de $\mathbf{9 0 \%}$ das prostatectomias radicais nos EUA são realizadas com assistência robótica e mais de 5 milhões de pacientes já foram operados com auxilio robótico em todo o mundo. A PRR traz os benefícios da cirurgia minimamente invasiva em relação à dor pós-operatória, menor perda de sangue, menor tempo de hospitalização, recuperação mais rápida em comparação com a cirurgia aberta, sem perder, entretanto, a eficácia no controle do câncer e da preservação das funções urinárias e sexuais.

Palavras-chave: Câncer de Próstata; Prostatectomia; Assistência Robótica.

Individuals diagnosed with prostate cancer often have to choose if they undergo the surgical treatment, which could influence the quality of their lives. Radical prostatectomy can impact cancer control and sexual and urinary functions in the patient, despite being the main form of curative treatment for localized prostate cancer. In general, patients select less morbid and minimally invasive treatments since the treatments have equivalent effectiveness. Robotic radical prostatectomy (RRP) represents the latest breakthrough in the surgical treatment of prostate cancer. This treatment is a minimally invasive surgical approach to laparoscopic prostatectomy, optimized by robotic technology. The use of robotic arms with instruments that perform movements with the same amplitude as human wrist, and a tremor filter, combined with an advanced three-dimensional imaging system, allowed the surgeon to operate, dissect, suture with a refinement of movement and quality of the imaging. Current estimates show that over $90 \%$ of radical prostatectomies in the United States are performed with robotic assistance, and more than 5 million patients have been 
operated by robotic assistance worldwide. RRP brings the benefits of minimally invasive surgery concerning postoperative pain, less blood loss, shorter hospital stay, faster recovery compared to open surgery, without losing the effectiveness in the cancer control, besides preserving the of urinary and sexual functions.

Keywords: Prostate Cancer; Prostatectomy; Robotic Assistance.

\section{Introdução}

O câncer de próstata é a neoplasia maligna mais comum em homens e a segunda causa de morte relacionada ao câncer. ${ }^{1}$ Indivíduos diagnosticados com câncer de próstata muitas vezes devem escolher se irão se submeter ao tratamento, o que provavelmente irá influenciar na qualidade de suas vidas. Tratamentos radicais com objetivo curativo consistem em prostatectomia radical (PR) e radioterapia. A cirurgia representa a principal forma de tratamento curativo do câncer de próstata nãometastático e é tipicamente oferecida a pacientes com câncer de próstata localizado (estágio $\leq$ T2) e expectativa de vida superior a 10 anos. ${ }^{2}$ Pacientes com fatores de pior prognóstico, tais como doença localmente avançada (estágio $>$ T3a), escore de Gleason $>8$ e nível de PSA $>20 \mathrm{ng} / \mathrm{mL}$, também podem ser beneficiados, porém, eles provavelmente precisarão associar outros tratamentos à cirurgia. ${ }^{3}$

A prostatectomia radical (PR) envolve a remoção da próstata e dos tecidos circundantes e das vesículas seminais, seguido de reconstrução através de uma anastomose entre a uretra e o colo da bexiga, e pode ser realizada através de uma das quatro abordagens cirúrgicas:

1. Via retropúbica aberta: a operação é realizada através de uma incisão primária através da área pubiana;

2. Via perineal aberta: a operação é realizada através de uma incisão primária no períneo (uma abordagem raramente realizada em comparação com a prostatectomia retropúbica aberta);

3. Laparoscopia "convencional": trata-se de uma abordagem minimamente invasiva, na qual a operação é realizada através de pequenas incisões no abdome; uma câmera de vídeo é inserida para ajudar o cirurgião para ver a próstata;

4. Laparoscopia assistida por robô: é semelhante à abordagem laparoscópica; no entanto, um cirurgião manipula os braços robóticos de um sistema cirúrgico que são inseridos através de pequenas incisões no abdome e utiliza um sistema de imagem tridimensional de alta definição.

\section{Evolução da Técnica}

Apesar de ser a principal forma de tratamento curativo para o câncer de próstata localizado, a PR pode impactar no controle do câncer, mas também na função sexual e urinária de cada individuo. ${ }^{2} \mathrm{Em}$ geral, os pacientes selecionam tratamentos menos mórbidos e minimamente invasivos desde que apresentem eficácias equivalentes. Dependendo das características do tumor e da função sexual do paciente, a PR pode ser realizada com preservação do feixe vásculonervoso adjacente à próstata (para preservar a potencia sexual) ou, sem esta preservação, em casos de doença localmente avançada ou em pacientes previamente impotentes. A linfadenectomia pélvica (remoção de linfonodos na pelve) pode ser realizada concomitantemente e é geralmente reservada para pacientes com maior risco de acometimento linfonodal. ${ }^{3}$

A prostatectomia radical robótica (PRR) representa o mais recente avanço no tratamento cirúrgico do câncer de próstata. Ele se baseia na abordagem cirúrgica minimamente invasiva da prostatectomia laparoscópica, otimizada pela tecnologia robótica. ${ }^{4}$

Um século passou desde a primeira prostatectomia para tratar câncer de próstata por via perineal, porém apenas na década de 1980 o melhor detalhamento anatômico permitiu a sua realização com mais segurança e menor 
morbidade. ${ }^{5}$ Estatécnicadeprostatectomiaradical aberta, por via retropúbica, com preservação do feixe neurovascular tornou-se o padrão no tratamento cirúrgico do câncer de próstata nãometastático. Na busca de uma via de acesso menos invasiva, Schuessler e colaboradores ${ }^{6}$ realizaram pela primeira vez a PR laparoscópica na década de 90 , mas não conseguiram demonstrar vantagens em relação à prostatectomia aberta, e o procedimento foi abandonado. Mais adiante, na França, Guillonneau e Vallancien ${ }^{7}$ descreveram e validaram suas técnicas de prostatectomia laparoscópica trazendo novo interesse no tratamento cirúrgico minimamente invasivo do câncer de próstata. Vários centros relataram resultados favoráveis a curto e médio prazos sobre os resultados funcionais e oncológicos da prostatectomia laparoscópica. ${ }^{8}$ Entretanto, a prostatectomia laparoscópica não foi amplamente adotada pela comunidade urológica, principalmente devido à dificuldade de aprendizagem e aos desafios técnicos relacionados. ${ }^{9}$ A incorporação de equipamentos robóticos sofisticados como o Sistema da Vinci (Intuitive Surgical, Inc.), trouxeram um novo alento ao permitirem uma redução significativa do tempo operatório assim como reduziram o tempo da curva de aprendizado. A utilização de braços robóticos e instrumentos capazes de realizar movimentos com a mesma amplitude do punho humano, com filtro para tremores, associado a um sistema avançado de imagem tridimensional, permitiram ao cirurgião operar, dissecar, suturar com um refinamento de movimentos e qualidade de imagem sem precedentes.

Em 2002, o trabalho pioneiro do Vattikuti Urology Institute, em Detroit, Michigan, abriu o caminho para uma nova era na PRR ao demonstrar sua técnica em mais de 1.000 pacientes operados. ${ }^{10,11}$

Desde então, um número crescente de instituições em todo o mundo tem utilizado a prostatectomia robótica como principal forma de tratamento cirúrgico do câncer de próstata. Estimativas atuais dão conta de que mais de
$90 \%$ das prostatectomias radicais nos EUA, são realizadas com assistência robótica e mais de 5 milhões de pacientes já foram operados com auxilio robótico em todo o mundo. ${ }^{12}$ Fica claro que a cirurgia assistida por robô já percorreu um longo caminho e desenvolveu-se em uma técnica usada em todo o mundo. No entanto, não se tornou "cirurgia robótica" no verdadeiro sentido do termo; não há inteligência de máquina fazendo cirurgia por conta própria e isso certamente não está à vista. Também não é uma telecirurgia operada por humanos. Atrasos mínimos na transmissão de longa distância tornaram impossível implementar a verdadeira telecirurgia. Em vez disso, a cirurgia assistida por robô é uma cirurgia laparoscópica refinada, com o sistema robótico proporcionando melhor visão, melhor controle do instrumento, dissecção mais refinada e melhor ergonomia para o cirurgião. ${ }^{13}$

O sistema cirúrgico da Vinci (Intuitive Surgical Inc. - Figuras 1 e 2) incorpora quatro braços robóticos sendo um braço controlando a ótica e os demais braços controlando instrumentos (tecnologia EndoWrist). Neste sistema, o robô é controlado pelo cirurgião que está confortavelmente sentado no console operacional a poucos metros de distância do paciente. A manipulação dos controles é transmitida para um computador que filtra, escala e transmite os movimentos do cirurgião para os braços e instrumentos robóticos, eliminado completamente o tremor fisiológico. Não há atraso mensurável entre o movimento dos controles no console e o movimento dos instrumentos dentro do paciente. Osinstrumentos com sistema EndoWrist apresentam-se como se tivessem verdadeiros punhos nas suas extremidades, permitindo grande amplitude em seu movimento, simulando a mão humana. Com visão $3 \mathrm{D}$, o sistema cirúrgico Da Vinci ajuda o cirurgião a identificar mais facilmente estruturas delicadas, como nervos e vasos sanguíneos ao redor da próstata. Os instrumentos EndoWrist fornecem ao cirurgião a destreza não disponível nos instrumentos laparoscópicos convencionais. 


\section{Influência da Técnica}

Apesardosavançosnoconhecimentoanatômico e no aprimoramento técnico, a prostatectomia radical está associada a eventos adversos de longo prazo. A continência completa, definida como nenhuma necessidade de proteção, varia entre $80 \%$ e $97 \%$ após a prostatectomia radical aberta. ${ }^{14}$ O retorno da função erétil varia entre $13 \%$ e $68 \%$ para a preservação do nervo unilateral e $32 \%$ a $86 \%$ para a preservação bilateral do nervo. ${ }^{14}$

Esses resultados também são afetados pelo método de coleta de dados. Por exemplo, informações baseadas em entrevistas com os pacientes tendem a apresentar resultados superestimados quando comparados com aqueles obtidos através de questionários validados preenchidos pelos próprios pacientes.

Desde a descrição por Walsh ${ }^{5}$ da abordagem anatômica da prostatectomia retropúbica radical, tornou-se evidente que a técnica cirúrgica é importante não apenas para o controle do câncer, mas também se correlaciona ao resultado funcional na medida que consegue uma maior preservação das funções sexuais e do controle urinário. As cirurgias minimamente invasivas trazem benefícios em relação à dor pós-operatória, pouca perda de sangue, melhores resultados cosméticos e recuperação mais rápida em comparação com a cirurgia aberta. ${ }^{15}$

Epstein e colaboradores ${ }^{16}$ relataram que a sobrevida livre de progressão após 10 anos é cerca de $25 \%$ maior em homens com margens negativas quandocomparadosàquelescommargenspositivas (79\% x 55\%). Ficou demonstrado que a técnica cirúrgica é um preditor independente do status da margem cirúrgica, ${ }^{17}$ que é positiva, em média, em $28 \%$ dos pacientes após a prostatectomia radical aberta. ${ }^{18}$ Como o sistema robótico aprimora as habilidades técnicas dos cirurgiões, ele pode oferecer o potencial da remoção mais precisa do câncer (margens negativas na doença confinada ao órgão) e melhor preservação da função sexual e do controle urinário.

A maioria dos cirurgiões usa uma abordagem transperitoneal, mas a técnica de PRR extraperitoneal tem a vantagem de não romper a cavidade peritoneal e qualquer vazamento anastomótico estará contido no espaço extraperitoneal. No entanto, devido a este espaço limitado, a cirurgia é dificultada e o trabalho de assistente torna-se mais desafiador. ${ }^{19}$

\section{Curva de Aprendizagem}

A curva de aprendizagem de uma nova técnica é sempre uma questão de debate, especialmente durante as fases iniciais de validação. Embora a cirurgia robótica utilize tecnologia complexa, a interface mecânica no console do cirurgião fornece transferência quase-intuitiva de habilidades cirúrgicas abertas. No caso da prostatectomia radical, há um consenso de que a curva de aprendizagem é mais rápida na cirurgia robótica quando comparada à laparoscopia convencional. ${ }^{20}$ Isso tem um grande impacto na medida que os melhores resultados chegarão mais precocemente, reduzindo assim as complicações em um número significativo de pacientes. Ahlering e colaboradores ${ }^{21}$ demonstraram a necessidade de apenas 12 cirurgias robóticas para que a curva de aprendizagem para a proficiência de 4 horas de cirurgia em uma prostatectomia radical e o tempo médio de operação fosse de 3,45 horas (variando de 2,5 a 5,1 horas) para a coorte inicial de 45 pacientes.

Menon e colaboradore ${ }^{15}$ estimaram que a curva de aprendizagem da PRR foi de aproximadamente 18 casos, a fim de alcançar um nível comparável de eficiência como cirurgiões experientes em prostatectomia laparoscópica.

\section{Prostatectomia Robótica versus Prostatectomia Aberta}

Os objetivos gerais da prostatectomia radical assistida por robô são manter os benefícios da cirurgia minimamente invasiva e, ao mesmo tempo, maximizar:

- Controle do câncer;

- Continência urinária - a capacidade de controlar voluntariamente a liberação de urina; 
Figura 1. Console, braços robóticos e sistema de vídeo do robô cirúrgico Da Vinci, Intuitive, Inc.

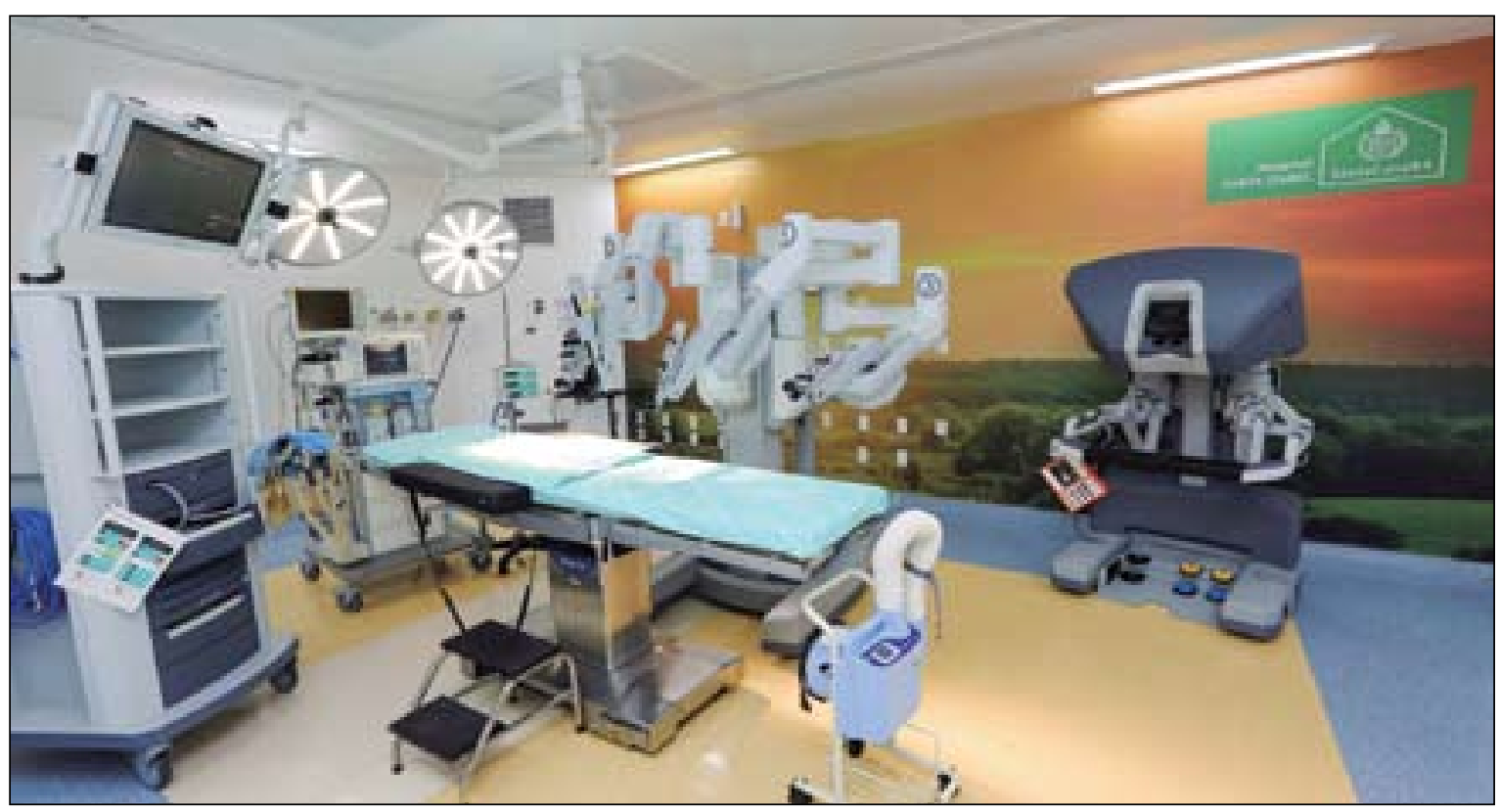

Figura 2. Prostatectomia radical robótica em curso.

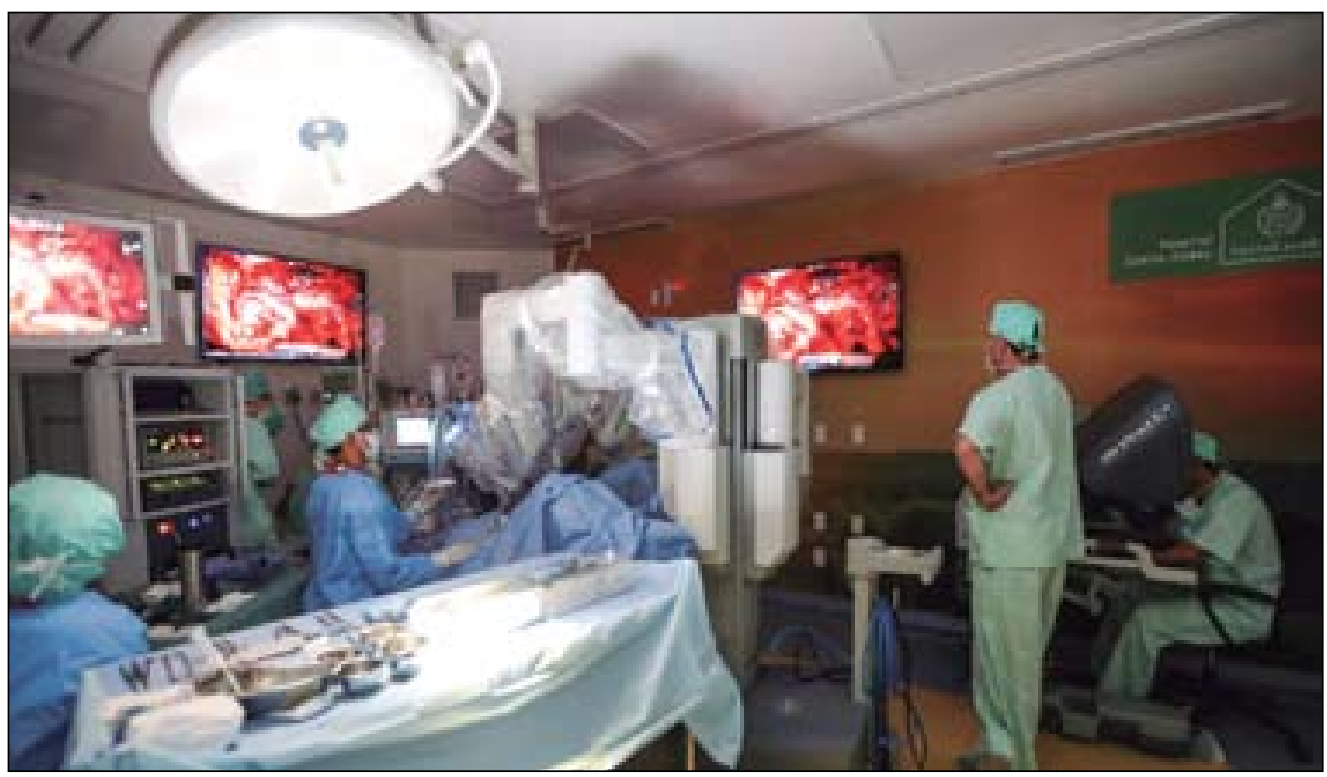

- Função erétil - a capacidade de manter um pênis ereto durante a atividade sexual.

Em comparação com a abordagem retropúbica aberta, que é realizada através de uma incisão de 10 a $15 \mathrm{~cm}$, a prostatectomia radical assistida por robô envolve múltiplas incisões que variam de 5 a $12 \mathrm{~mm}$ de diâmetro.
A preferência do paciente é essencial ao processo de tomada de decisão para o tratamento do câncer de próstata. Apesar de ter sido motivo de muitos debates, a comparação atual da técnica robótica com as demais técnicas já é considerada uma questão ultrapassada, uma vez que o mundo inteiro já adota a técnica robótica como padrão- 
ouro no tratamento cirúrgico do câncer de próstata. De qualquer forma, é importante apresentar importantes trabalhos que respaldam o resultado que vemos no nosso cotidiano. Em geral, os pacientestendemapreferiranaturezaminimamente invasiva da prostatectomia radical assistida por robô em comparação com a prostatectomia radical aberta, porque há menos cicatrizes cirúrgicas, menor tempo de hospitalização e uma recuperação mais rápida. ${ }^{22,23}$

Com base nos dados dos estudos acima, pacientes com maior nível socioeconômico são mais propensos às opções minimamente invasivas (laparoscópica ou assistida por robô). Outro aspecto que converge para maior interesse pelos pacientes, a cobertura da mídia e o marketing online para prostatectomia radical assistida por robô também são mais difundidos apesar de existir o potencial para informações imprecisas de fontes online. ${ }^{24,25}$ Esta convergência em direção à tecnologia mais avançada traz ao paciente a sensação de estar sendo submetido a um procedimento mais simples, com menos riscos. Esta é a provável explicação para eventual arrependimento de alguns pacientes submetidos à prostatectomia robótica, possivelmente porque suas expectativas eram muito altas conforme foi identificado em um estudo conduzido nos Estados Unidos. ${ }^{26}$ Já o estudo canadense feito por Davison e colaboradores, que avaliaram o arrependimento de decisão usando a Decision Regret Scale para medir sofrimento e arrependimento após um ano de uma determinada cirurgia, demonstraram escores baixos de arrependimento tanto no grupo de cirurgia robótica quanto no grupo de cirurgia aberta. $^{27}$

Do ponto de vista de análise da eficácia e das possíveis vantagens na comparação entre as diferentes vias de acesso, devem-se considerar as seguintes questões: quais são os benefícios e malefícios da prostatectomia radical assistida por robô para o câncer de próstata, em comparação com a prostatectomia radical aberta e laparoscópica radical e laparoscópica convencional (sem assistência robótica)? Esta análise deve contemplar diferentes desfechos, tais quais:

- Desfechosperioperatórios:tempocirúrgico; tempo de internação hospitalar; perda de sangue estimada; taxas de transfusão sanguínea; tempo de permanência da sonda vesical; taxas de complicações;

- Desfechos funcionais: taxa de incontinência urinária; taxa de impotência sexual; dor pós-operatória; tempo de convalescença.

- Desfechos oncológicos: taxas de margens cirúrgicas positivas; taxas de recorrência do câncer.

\section{Tempo Operatório}

Foram utilizadas diferentes definições de "tempo operatório", do tempo operatório total ao tempo pele-a-pele (ou seja, o tempo desde a incisão até a sutura final). No entanto, apesar das diferenças na medição, a tendência geral foi uma diminuição significativa no tempo operatório em favor da abordagem aberta nos estudos não randomizados, o que pode ser explicado pelo tempo extra de preparação necessário para a abordagem assistida por robô. Em contraste, o estudo randomizado de Yaxley e colaboradores ${ }^{28}$ mostrou um aumento significativo no tempo operatório para a abordagem aberta (tanto para a duração da cirurgia quanto para o tempo de sala operatória). Obviamente, também existe o impacto da curva de aprendizagem associado ao tempo operatório e à experiência do cirurgião. Doumerc e colaboradores ${ }^{29}$ observaram que o tempo operatório para prostatectomia radical assistida por robô diminuiu com a experiência e continuou a diminuir durante o período do estudo, com cerca de 110 casos necessários para atingir uma duração operatória de menos de 3 horas.

\section{Tempo de Hospitalização}

ATabela 1 mostra o tempo de hospitalização em pacientes submetidos a PRR e PR convencional em diversos estudos. 


\section{Perda de Sangue Estimada}

Diversos estudos avaliaram a perda sanguínea estimada para prostatectomia radical assistida versus cirurgia aberta. Todos os estudos mostraram perda sanguínea significativamente menor estimada para prostatectomia radical assistida por robô (Tabela 2). Houve grandes variações na perda de sangue entre os grupos, variando de $100 \mathrm{~mL}$ para mais de $400 \mathrm{~mL}$ para cirurgia assistida por robô e, em torno de $500 \mathrm{~mL}$ a mais de $1.300 \mathrm{~mL}$ nas cirurgias abertas.

Fode e colaboradores descobriram que, juntamente com um baixo volume prostático ( $\mathrm{p}$ $<0,001$ ), cirurgia sem preservação nervosa ( $p$ $<0,001)$ e cirurgião $(\mathrm{p}<0,001)$, a prostatectomia radical assistida por robô foi um preditor de baixa perda sanguínea perioperatória na análise multivariada. Houve um risco relativo de 2,89 (intervalo de confiança de 95\% [IC] 2,52-3,3, $\mathrm{p}<0,001)$ entre os grupos aberto e assistido por robô. ${ }^{32}$

\section{Duração do Cateterismo Vesical}

Geralmente, menores durações foram encontradas para o grupo de prostatectomia radical assistida por robô, com um intervalo de 3 a 12 dias para cirurgia assistida por robô e 6 a 11 dias para cirurgia aberta. A ampla gama de resultados provavelmente é devido a diferentes protocolos hospitalares e procedimentos para o tempo de remoção.

Di Pierro e colaboradores ${ }^{36}$ mediram as taxas livres de cateter nos dias 10 e 15 e enquanto as taxas favoreceram o grupo assistido por robô, nenhum valor de $\mathrm{p}$ foi relatado para mostrar significância. Este estudo foi um dos dois únicos estudos que avaliaram o cateterismo prolongado ( $>10$ dias após a cirurgia), sem diferença significativa entre procedimentos assistidos por robô e procedimentos abertos. Doumerc e colaboradores ${ }^{29}$ também observaram o cateterismo prolongado no grupo aberto; no entanto, não relataram valores para significância.
Em contraste com os estudos não randomizados, que relataram durações significativamente mais curtas para o tempo de permanência do cateterismo durante a prostatectomia radical assistida por robô, no estudo controlado randomizado recentemente publicado não houve diferença entre os grupos. $^{28}$

\section{Complicações Pós-Operatórias}

Carlsson e colaboradores ${ }^{37}$ examinaram especificamente as complicações na prostatectomia radical assistida por robô e aberta. As complicações de Clavien grau IIIb a grau $\mathrm{V}$ (mais graves) foram mais comuns no grupo aberto $(12,9 \%)$ do que no grupo assistido por robô $(3,7 \%)$. Entre as complicações pósoperatórias tardias ( $>30$ dias a 15 meses), eles descobriram que as contraturas do colo da bexiga foram tratadas mais frequentemente no grupo aberto $(4,5 \%)$ do que no grupo assistido por robô $(0,2 \%)(p<0,001)$. Além disso, mais pacientes precisaram de cirurgia por causa da incontinência pós-operatória no grupo aberto $(2,2 \%$ versus $0,5 \%$ assistido por robô, $\mathrm{p}<0,01) .{ }^{37}$

\section{Função Urinária}

Um grupo de pesquisadores de Ontario no Canadá ${ }^{38}$ realizou uma metanálise de estudos que compararam a função urinária após cirurgia robótica e aberta. As definições para desfechos dicotômicos foram principalmente o uso de fraldas ou ausência de vazamento, com definições ligeiramente diferentes entre os estudos, de estritamente sem absorventes ( 0 pads) a 0 ou 1 absorvente por dia. Segundo o resultado da metanálise, quatro estudos relataram tempo para continência, sendo três estudos mostrando recuperação de continência significativamente mais rápida com prostatectomia radical assistida por robô. ${ }^{38}$

Após correção para covariáveis, Geraerts e colaboradores ${ }^{39}$ acharam que a diferença no tempo para a continência era significativa 
(razão de risco 1,522, IC95\% 1,027-2,255, p = $0,036)$. Além disso, homens mais jovens, homens com margens cirúrgicas positivas e homens sem incontinência pré-operatória alcançaram a continência mais cedo. Uma comparação do tempo até a continência entre os grupos com um número suficiente de pacientes (risco intermediário e/ou bilateral) ainda mostrou um retorno mais rápido da continência após a prostatectomia radical assistida por robô, mas o efeito foi menor e não significativo (razão de risco $>1,2, p>0,05$ ). $\mathrm{O}$ grupo assistido por robô também teve significativamente menos sintomas de micção do que o grupo aberto em 1 mês $(p=0,01)$ e 3 meses $(p=0,04)$ após a cirurgia. Aos 12 meses, os pacientes do grupo aberto foram mais limitados fisicamente e tomaram mais precauções para evitar um vazamento de urina do que aqueles no grupo assistido por robô $(p=0,01$ e $\mathrm{p}=0,01$, respectivamente).

\section{Função Erétil}

Este mesmo grupo canadense, ${ }^{38}$ realizou uma metanálise de estudos que compararam a função erétil após cirurgia robótica e aberta. Os resultados foram determinados principalmente por uma única pergunta: se os pacientes tinham ereções suficientemente firmes para a relação sexual. Um estudo mostrou que o tempo para a potência foi significativamente menor no grupo assistido por robô. ${ }^{40}$

Considerando apenas os pacientes submetidos à cirurgia bilateral poupadora de nervos, com 12 meses ou mais de acompanhamento, Ficarra e colaboradores $^{41}$ descobriram que $49 \%$ no grupo aberto e $81 \%$ no grupo assistido por robô eram potentes $(\mathrm{p}<0,001)$. Da mesma forma, ao avaliar apenas pacientes com idade $<65$ anos e com escore de Charlson $\leq 2$, descobriram que $58 \%$ no grupo aberto e $84 \%$ no grupo assistido por robô eram potentes $(p<0,01)$. Pacientes que tiveram um ou dois nervos poupados relataram um escore de qualidade de vida sexual médio global maior do que aqueles com nenhum nervo (ou desconhecido) poupado. Resultados semelhantes foram observados para pacientes que usaram um inibidor de PDE5 versus pacientes sem uso de inibidores da PDE5. ${ }^{41}$

Fode e colaboradores ${ }^{32}$ encontraram, na análise univariada, que não houve diferença estatisticamente significante entre os grupos nas proporções de pacientes potentes em 3 e 12 meses $(p=0,08$ e $p=0,16$, respectivamente). No entanto, significativamente mais pacientes no grupo assistido por robô haviam recuperado a potência aos 6 meses $(p=0,02)$.

\section{Dor}

$\mathrm{O}$ estudo randomizado capitaneado por Yaxley ${ }^{28}$ observou redução significativa da dor a curto prazo, favorecendo a prostatectomia radical assistida por robô às 24 horas e 1 semana póscirurgia. Esses resultados foram relacionados àdor durante as atividades e quando experimentaram a pior dor, não durante o descanso.

\section{Taxas de Margens Cirúrgicas Positivas}

Quando analisamos os resultados pelas taxas de margem cirúrgica positivas totais, encontramos uma heterogeneidade considerável dentre diversos estudos.

Doumerc e colaboradores ${ }^{29}$ investigaram o efeito da curva de aprendizado (experiência do cirurgião). Eles descobriram que a taxa geral de margens cirúrgicas positivas para cirurgia assistida por robô diminuiu à medida que a experiência do cirurgião aumentou. Um efeito da curva de aprendizado também foi observado nos cânceres estágio pT2. No entanto, as taxas entre pT2 e pT3 permaneceram estatisticamente diferentes no final do estudo. Os autores observaram que isso pode ter ocorrido devido ao baixo número de casos disponíveis para análise ou porque a curva de aprendizagem ainda não havia sido superada.

Fode e colaboradores, ${ }^{32}$ utilizando análises univariadas, também examinaram a curva de aprendizagem. Eles descobriram que, comparada à prostatectomia assistida por robô, 
Tabela 1. Tempo de permanência em dias no hospital.

\begin{tabular}{llccc}
\hline Autor, ano & Medida & \multicolumn{2}{c}{ Tempo de permanência em dias } & Valor p \\
\cline { 3 - 4 } & & Robô-Assistida & Cirurgia Aberta & \\
\hline Estudo Controlado Randomizado & Média & $1,6 \pm 2,6$ & $3,3 \pm 1,5$ & $<, 001$ \\
$\begin{array}{llccc}\text { Yaxley e colaboradores (2016) } \\
\text { Estudos Não-Randomizados }\end{array}$ & & & & \\
Doumerc e colaboradores (2010) & Média & $2,8 \pm 2,4$ & $5,5 \pm 2,7$ & $<, 001$ \\
Philippou e colaboradores $(2012)^{30}$ & Média & $1,3(1-3)$ & $3,8(2-7)$ & $<, 001$ \\
Haglind e colaboradores $(2015)^{31}$ & Mediana & $3(2-4)$ & $4(3-5)$ & $<, 001$ \\
\hline
\end{tabular}

Tabela 2. Estimativa de perda de sangue para prostatectomia robô-assistida versus cirurgia aberta.

\begin{tabular}{|c|c|c|c|c|}
\hline \multirow[t]{2}{*}{ Autor, Ano } & \multirow[t]{2}{*}{ Medida } & \multicolumn{2}{|c|}{ Perda estimada de sangue, $\mathrm{mL} \pm \mathrm{DP}$} & \multirow[t]{2}{*}{ Valor $\mathrm{p}$} \\
\hline & & Robô-Assistida & Cirurgia Aberta & \\
\hline Estudo Controlado Randomizado & & & & \\
\hline $\begin{array}{l}\text { Yaxley e colaboradores }(2016)^{28} \\
\text { Estudos Não-Randomizados }\end{array}$ & Média & $444 \pm 294$ & $1,338 \pm 591$ & $<, 001$ \\
\hline Hong e colaboradores $(2010)^{33}$ & Média & $457 \pm 281$ & $1410 \pm 901$ & $<, 05$ \\
\hline Philippou e colaboradores $(2012)^{30}$ & Média & $132 \pm 151$ & $513 \pm 343$ & $<, 001$ \\
\hline Bier e colaboradores $(2016)^{34}$ & Mediana & $400(100-1.300)$ & $700(234-1.600)$ & $<, 001$ \\
\hline Fode e colaboradores $(2014)^{32}$ & Mediana & $150(5-1.500)$ & $600(50-4.320)$ & $<, 001$ \\
\hline Lott e colaboradores $(2015)^{35}$ & Mediana & $212(50-1.200)$ & $487(150-1.250)$ & 01 \\
\hline Haglind e colaboradores $(2015)^{31}$ & Média & $100(50-200)$ & $550(350-800)$ & $<, 001$ \\
\hline
\end{tabular}

a prostatectomia radical aberta tinha um risco relativo de margens cirúrgicas positivas de 1,56 (95\% IC 1,23-1,99, $\mathrm{p}<0,001)$. No entanto, nas análises multivariadas, com estratificação das características tumorais, das características do paciente e do cirurgião, o tipo de cirurgia não afetou as margens cirúrgicas $(\mathrm{p}=0,96)$. Um grande volume tumoral $(\mathrm{p}<0,001)$, estágio tumoral patológico $(\mathrm{p}=0,005)$ e um pequeno volume prostático $(\mathrm{p}=0,04)$ foram preditores independentes de margens cirúrgicas positivas.

Thompson e colaboradores $^{42}$ também examinaram a curva de aprendizagem e descobriram que, na doença $\mathrm{T} 2$, as chances de uma margem cirúrgica positiva eram inicialmente maiores, porém, no final do estudo, após 866 casos, as chances de uma margem cirúrgica positiva foram $55 \%$ menores para a cirurgia assistida por robô em comparação com a abordagem aberta (OR 0,45, IC 95\% 0,22-0,92).

\section{Taxas Bioquímicas sem Recidiva}

Três estudos não randomizados relataram taxas livres de recidiva bioquímica (a ausência de níveis elevados de PSA após a prostatectomia radical). Di Pierro e colaboradores ${ }^{36}$ não encontraram diferença entre as taxas livres de recidiva bioquímica em 3 ou 12 meses, assim como Philippou e colaboradores, ${ }^{30} \mathrm{em} 12$ meses. Nenhum estudo relatou informações sobre as taxas de sobrevida livre de câncer. No estudo randomizado de Yaxley, ${ }^{28}$ resultados a longo prazo que incluem recorrência bioquímica ainda não foram publicados.

\section{Custos}

A questão dos custos em torno da cirurgia robótica ainda não foi resolvida. Guru e colaboradores $^{43}$ realizaram uma análise de custo 
para 30 pacientes consecutivos submetidos a PRR e 30, a cirurgia aberta.

Os custos gerais foram divididos em 9 categorias, dos quais laboratório e suprimentos foram maiores para a abordagem robótica; já farmácia, sala de recuperação e cuidados de ala foram maiores para a abordagem aberta; e anestesia e custos de sala operatória não foram estatisticamente diferentes. O custo total por caso, excluindo a manutenção e a depreciação do sistema robótico, foi semelhante para ambas as abordagens.

No entanto, do ponto de vista da saúde, a economia não calculada obtida na recuperação mais rápida do paciente, menor custo no tratamento das complicações e no retorno à atividade e produtividade normais precisa ser considerada. Ademais, a chegada de novas plataformas robóticas sinalizam para possível redução dos custos do investimento e implantação do Programa de Robótica nos hospitais, o que certamente ajudará a diminuir ainda mais o custo final das cirurgias robóticas assistidas.

\section{Conclusões}

A evidência atual sugere que a PRR é comparável em eficácia à prostatectomia aberta e laparoscópica, apresentando vantagens em diferentes parâmetros clínicos, funcionais e patológicos, com tendência de redução de custos de implantação e manutenção.

\section{Referências}

1. Jemal A, Murray T, Samuels A et al. Cancer Statistics, 2003. CA Cancer J Clin. 2003;53:5-26.

2. Bill-Axelson A, Holmberg L, Ruutu M et al. Radical prostatectomy versus watchful waiting in early prostate cancer. SPCG-4 Investigators. N Engl J Med. 2011 May 5; 364(18):1708-17.

3. Allaf ME, Partin AW, Carter HB. The importance of pelvic lymph node dissection in men with clinically localized prostate cancer. Rev Urol. 2006;8(3):112-119.

4. Abbou CC, Hoznek A, Salomon L et al. Laparoscopic radical prostatectomy with a remote-controlled robot. J Urol. 2001; 165:1964-1966.
5. Walsh PC, Lepor H, Eggleston JC. Radical prostatectomy with preservation of sexual function: anatomical and pathological considerations. Prostate. 1983;4:473-85.

6. Schuessler WW, Schulam PG, Clayman RV et al. Laparoscopic radical prostatectomy: initial short-term experience. Urology. 1997; 50:854-857.

7. Guillonneau B, Vallancien G. Laparoscopic radical prostatectomy: the Montsouris technique. J Urol. 2000; 163:1643-9.

8. Rassweiler J, Schulze M, Teber D et al. Laparoscopic radical prostatectomy: functional and oncological outcomes. Curr Opin Urol. 2004;14:75-82.

9. Kavoussi LR. Laparoscopic radical prostatectomy: irrationalexuberance? Urology. 2001;58:503-5.

10. Menon M, Shrivastava A, Tewari A et al. Laparoscopic and robot assisted radical prostatectomy: establishment of a structured program and preliminary analysis of outcomes. J Urol. 2002;168:945-949.

11. Tewari A, Peabody J, Sarle R et al. Technique of da Vinci robot-assisted anatomic radical prostatectomy. Urology. 2002; 60:569-572.

12. https://www.intuitive.com/en-us/patients/patients. Acesso 18/08/2019.

13. Hakenberg OW. A brief overview of the development of robot-assisted radical prostatectomy. Arab J Urol. 2018 Jul 24;16(3):293-296.

14. Salomon L, Sebe P, De La Taille A et al. Open versus laparoscopic radical prostatectomy: Part II. BJU Int. 2004;94:244-250.

15. Menon M, Tewari A, Baize B et al. Prospective comparison of radical retropubic prostatectomy and robot-assisted anatomic prostatectomy: the Vattikuti Urology Institute experience. Urology. 2002;60:864-8.

16. Epstein JI, Partin AW, Sauvageot J et al. Prediction of progression following radical prostatectomy. A multivariate analysis of 721 men with long-term follow-up. Am J SurgPathol. 1996;20:286-292.

17. Eastham JA, Kattan MW, Riedel E et al. Variations among individual surgeons in the rate of positive surgical margins in radical prostatectomy specimens. J Urol. 2003;170:2292-5.

18. Wieder JA, Soloway MS. Incidence, etiology, location, prevention and treatment of positive surgical margins after radical prostatectomy for prostate cancer. J Urol. 1998;160:299-315.

19. Gettman MT, Hoznek A, Salomon L et al. Laparoscopic radical prostatectomy: description of the extraperitoneal approach using the da Vinci robotic system. J Urol. 2003;170:416-419.

20. El-Hakim A, Tewari A. Robotic Prostatectomy- A Review. Med Gen Med. 2004 Oct 25;6(4):20.

21. Ahlering TE, Skarecky D, Lee D et al. Successful transfer of open surgical skills to a laparoscopic environment using a robotic interface: initial experience with laparoscopic radical prostatectomy. J Urol. 2003;170:1738-1741. 
22. Xu J, Dailey RK, Eggly S, Neale AV, Schwartz KL. Men's perspectives on selecting their prostate cancer treatment. J Natl Med Assoc. 2011;103(6):468-78.

23. Rojo MA, Martinez-Salamanca JI, Maestro MA, Galarza IS, Rodriguez JC. Impact of cosmetic result on selection of surgical treatment in patients with localized prostate cancer. JSLS. 2014 OctDec;18(4):e2014.00024.

24. Steinberg PL, Ghavamian R. Searching robotic prostatectomy online: what information is available? Urology. 2011;77(4):941-5.

25. Mulhall JP, Rojaz-Cruz C, Muller A. An analysis of sexual health information on radical prostatectomy websites. BJU Int. 2010;105(1):68-72.

26. Schroeck FR, Krupski TL, Sun L, Albala DM, Price MM, Polascik TJ et al. Satisfaction and regret after open retropubic or robot-assisted laparoscopic radical prostatectomy. Eur Urol. 2008;54(4):785-93.

27. Davison BJ, Matthew A, Gardner AM. Prospective comparison of the impact of robotic-assisted laparoscopic radical prostatectomy versus open radical prostatectomy on health-related quality of life and decision regret. Can Urol Assoc J. 2014;8(12):E68-72.

28. Yaxley JW, Coughlin GD, Chambers SK, Occhipinti S, Samaratunga H, Zajdlewicz L, et al. Robotassisted laparoscopic prostatectomy versus open radical retropubic prostatectomy: early outcomes from a randomised controlled phase 3 study. Lancet. 2016;388(10049):1057-66.

29. Doumerc N, Yuen C, Savdie R, Rahman MB, Rasiah KK, Pe Benito R et al. Should experienced open prostatic surgeons convert to robotic surgery? The real learning curve for one surgeon over 3 years. BJU Int. 2010;106(3):378-84.

30. Philippou P, Waine E, Rowe E. Robot-assisted laparoscopic prostatectomy versus open: comparison of the learning curve of a single surgeon. J Endourol. 2012;26(8):1002-8.

31. Haglind E, Carlsson S, Stranne J, Wallerstedt A, Wilderang $\mathrm{U}$, Thorsteinsdottir $\mathrm{T}$, et al. Urinary incontinence and erectile dysfunction after robotic versus open radical prostatectomy: a prospective, controlled, nonrandomised trial. Eur Urol. 2015;68(2):216-25.

32. Fode M, Sonksen J, Jakobsen H. Radical prostatectomy: initial experience with robot-assisted laparoscopic procedures at a large university hospital. Scand J Urol. 2014;48(3):252-8.
33. Hong JY, Kim JY, Choi YD, Rha KH, Yoon SJ, Kil HK. Incidence of venous gas embolism during roboticassisted laparoscopic radical prostatectomy is lower than that during radical retropubic prostatectomy. $\mathrm{Br} \mathrm{J}$ Anaesth. 2010;105(6):777-81.

34. Bier S, Hennenlotter J, Rausch S, Aufderklamm S, Martzog JC, Stenzl A et al. Return to work and normal daily life activity after open and robot-assisted radical prostatectomy - a single surgeon analysis. Urol Int. 2016;96(3):280-6.

35. Lott FM, Favorito LA. Is previous experience in laparoscopic necessary to perform robotic radical prostatectomy? A comparative study with robotic and the classic open procedure in patients with prostate cancer. Acta Cir Bras. 2015;30(3):229-34.

36. Di Pierro GB, Baumeister P, Stucki P, Beatrice J, Danuser H, Mattei A. A prospective trial comparing consecutive series of open retropubic and robotassisted laparoscopic radical prostatectomy in a centre with a limited caseload. Eur Urol. 2011;59(1):1-6.

37. Carlsson S, Nilsson AE, Schumacher MC, Jonsson MN, VolzDS, Steineck G et al. Surgery-related complications in 1253 robot-assisted and 485 open retropubic radical prostatectomies at the Karolinska University Hospital, Sweden. Urology. 2010;75(5):1092-7.

38. Ont Health Technol Assess Ser. 2017;17(11):1-172.

39. Geraerts I, Van Poppel H, Devoogdt N, Van CleynenbreugelB, JoniauS, VanKampenM.Prospective evaluation of urinary incontinence, voiding symptoms and quality of life after open and robot-assisted radical prostatectomy. BJU Int. 2013;112(7):936-43.

40. Kim SC, Song C, Kim W, Kang T, Park J, Jeong IG et al. Factors determining functional outcomes after radical prostatectomy: robot-assisted versus retropubic. Eur Urol. 2011;60(3):413-9.

41. Ficarra V, Novara G, Fracalanza S, D’Elia C, Secco $\mathrm{S}$, Iafrate $\mathrm{M}$ et al. A prospective, non-randomized trial comparing robot-assisted laparoscopic and retropubic radical prostatectomy in one European institution. BJU Int. 2009;104(4):534-9.

42. Thompson JE, Egger S, Bohm M, et al. Superior quality of life and improved surgical margins are achievable with robotic radical prostatectomy after a long learning curve: a prospective single-surgeon study of 1552 consecutive cases. Eur Urol. 2014;65(3):521-31.

43. Guru K, Bhabdari A, Peabody J, et al. Cost comparison between robotic-assisted laparoscopic prostatectomy (Vattikuti Institute Prostatectomy) and radical retropubic prostatectomy. J Urol. 2004;171(4):43. 